\title{
Shortened single-walled nanotubes functionalized with poly(ethylene glycol): preparation and properties
}

\author{
Enzo Menna, ${ }^{a}$ Gianfranco Scorrano, ${ }^{a}$ Michele Maggini, ${ }^{a}$ Matteo Cavallaro, ${ }^{a}$ Federico \\ Della Negra, ${ }^{a}$ Marino Battagliarin, ${ }^{b}$ Renato Bozio, ${ }^{c}$ Fabiana Fantinel, ${ }^{c}$ and Moreno \\ Meneghetti $^{c}$ \\ ${ }^{a}$ ITM-CNR - Sezione di Padova, Dipartimento di Chimica Organica, Università di Padova, \\ Via Marzolo 1, I-35131 Padova, Italy \\ ${ }^{b}$ Dipartimento di Chimica Fisica, Università di Venezia, Via Torino 155/b, \\ I-30170 Venezia Mestre, Italy \\ ${ }^{c}$ Dipartimento di Chimica Fisica, Università di Padova, Via Loredan 2, \\ I-35131 Padova, Italy \\ E-mail:enzo.menna@unipd.it
}

\section{Dedicated to Professor Henry J. Shine on the occasion of his $80^{\text {th }}$ birthday}

(received 10 June 03; accepted 15 Sept 03; published on the web 30 Sept 03)

\begin{abstract}
Poly(ethylene glycol) (PEG) was grafted to shortened single-walled carbon nanotubes (shSWNTs) obtained from original SWNTs after acid oxidative cutting and etching processes. PEGsh-SWNTs, prepared by treating the sh-SWNTs with $\mathrm{SOCl}_{2}$ followed by amidation with PEGmonoamine (molecular weight $=2000$ ), are moderately soluble in tetrahydrofuran, chlorinated hydrocarbons (ca. $1 \mathrm{mg} / \mathrm{ml}$ ), sparingly soluble in water, acetone $\mathrm{CH}_{3} \mathrm{OH}$ and insoluble in non polar solvents such as toluene, hexanes or carbon disulfide. Non linear transmission measurements on solutions of PEG-sh-SWNTs in $\mathrm{CHCl}_{3}$ showed a better optical limiting performance relative to that recorded for original SWNTs suspended in the same solvent.
\end{abstract}

Keywords: Carbon nanotubes, optical limiting, polyethylene-glycol, dissolution

\section{Introduction}

Since the discovery of carbon nanotubes (NTs) in the early $1990 \mathrm{~s},{ }^{1}$ many theoretical and experimental studies have highlighted their peculiar behavior. ${ }^{2-4}$ In particular, SWNTs have attracted the attention of chemists and physicists because of their interesting electrical, optical, magnetic and mechanical properties. Potential applications were envisioned in the field of 
molecular electronics, ${ }^{5}$ composite polymers, ${ }^{6}$ nanowires, ${ }^{7}$ optical limiters, ${ }^{8,9}$ or gas-storage devices. $^{10}$

Unlike physical investigations of solid state properties of NTs, the study of their chemical reactivity is still in its infancy. An inherent problem associated to the development of a 'chemistry of nanotubes' lies in their insolubility in the solvents of common use that limits their chemical manipulation and wide application of their physical and electronic properties for practical uses. Several methods have been devised for the solubilization of NTs that could be roughly summarized in two categories: 1) suspension, ${ }^{11,12}$ polymer-wrapping, ${ }^{13-15}$ noncovalent sidewall functionalization ${ }^{16}$ and dissolution in aromatic amines ${ }^{17}$ of pristine NTs and 2) covalent functionalization of i) sh-SWNTs and shortened multi-walled nanotubes (sh-MWNTs) bearing carboxylic groups at the edges and ii) pristine SWNTs and MWNTs. Haddon and coworkers pioneered the approach of functionalizing the carboxylic groups of sh-SWNT via amidation with amines bearing long alkyl chains. ${ }^{18-21}$ A variety of soluble sh-SWNT derivatives have been prepared using this method also by other groups. ${ }^{22-38}$ The functionalization of pristine SWNTs with fluorine, alkanes, nitrenes, carbenes, radicals species and diazonium compounds was effective since relatively soluble materials could be obtained. ${ }^{39,40}$ Recently, Prato and coworkers successfully applied the 1,3-dipolar cycloaddition of azomethine ylides for the organic functionalization of pristine SWNTs and MWNTs which has led to a high level of solubility of the resulting products. ${ }^{41}$ It is worth mentioning, however, that an extensive sidewall covalent functionalization of original NTs may lead to partial or complete loss of the NTs original properties. $^{42}$

In 2001 Sano and coworkers described the synthesis and self-organization properties of PEGsh-SWNTs $\left(\mathrm{MW}_{\mathrm{PEG}}=5000\right)$ demonstrating how SWNTs segments conformation can be controlled by solvent quality as if they were ordinary hydrocarbon-based block copolymers. ${ }^{25}$ We recently reported a novel procedure for the microwave-assisted synthesis of PEG-shSWNTs. $^{38}$

In this paper we report the preparation of soluble PEG-sh-SWNTs $\left(\mathrm{MW}_{\mathrm{PEG}}=2000\right)$ and a preliminary study on their optical limiting performance in $\mathrm{CHCl}_{3}$ solutions relative to that of SWNTs suspended in the same solvent.

\section{Results and Discussion}

Following the Haddon amidation chemistry ${ }^{18}$ we grafted PEG chains to sh-SWNTs bearing carboxylic groups (SWNT-CO ${ }_{2} \mathrm{H}$ ), made from SWNTs cut and etched in acids according to the procedure of Smalley and coworkers. ${ }^{43}$ We selected PEG for its well-known solubilizing properties $^{44}$ and also in view of a possible beneficial effect of PEG chains in minimizing aggregation of NTs or of bundles of NTs. The reduced sonication time $(8 \mathrm{~h})$ for the cutting process furnished sh-SWNTs whose powder Raman spectrum (not shown) displayed only minor differences if compared to that of the original SWNTs which was in line with the powder Raman 
spectra reported in the literature. ${ }^{45,46}$ Since a precise estimate of the size and size distribution of the shortened NTs was not pursued, the samples used for the subsequent reaction with thionyl chloride are best described as mixtures of NTs with different length and diameters. PEG-shSWNTs were produced by refluxing first SWNT- $\mathrm{CO}_{2} \mathrm{H}$ in neat $\mathrm{SOCl}_{2}$ for 24 hours, followed by treatment of the SWNT-COCl derivatives with excess of carefully dried, neat PEG-monoamine $(\mathrm{MW}=2000)$ at $90{ }^{\circ} \mathrm{C}$ for 96 hours under inert atmosphere. PEG-sh-SWNTs are moderately soluble in a variety of weakly polar and polar organic solvents, such as THF and chlorinated hydrocarbons, but they are sparingly soluble in water, acetone, ethanol and essentially insoluble in toluene, hexanes or carbon disulfide. These results are in contrast with the findings of Sano and coworkers who reported a poor dispersion of their PEG-sh-SWNTs both in $\mathrm{CHCl}_{3}$ and THF. $^{25}$ In particular, their PEG-sh-SWNTs dispersions in $\mathrm{CHCl}_{3}$ collapse into globular aggregates. A possible explanation involves the different synthetic protocol used by Sano for grafting the PEG chains to SWNT-COCl although the origin of this major discrepancy is not easy to rationalize. Our solutions are homogeneous, strongly colored and are stable for weeks without any evident sedimentation. For example, the solubility of PEG-sh-SWNTs samples in $\mathrm{CHCl}_{3}$ at room temperature is about $1 \mathrm{mg} / \mathrm{ml}$, which was sufficient for non linear transmission measurements (vide infra).

In his paper, Sano and coworkers pointed out that unreacted PEG chains were difficult to remove even after repeated washings since they strongly entangle with the grafted chains. Therefore the possibility that our soluble materials contain both PEG-sh-SWNTs and physisorbed PEG chains cannot be ruled out. It is worth mentioning that $\mathrm{SWNT}-\mathrm{CO}_{2} \mathrm{H}$ are highly insoluble materials and that blends of SWNT- $\mathrm{CO}_{2} \mathrm{H}$ with PEG-amine did not give any soluble fraction containing NTs. This supports the view that the species in solution are mostly PEG-functionalized NTs.

FT-IR spectra of the PEG-sh-SWNTs exhibit, among others, a strong band at $1101 \mathrm{~cm}^{-1}$ which can be safely assigned to the CCO anti-symmetric stretching of the PEG chains, observed at $1117 \mathrm{~cm}^{-1}$ for the parent PEG-amine. The transition of the nanotube-bound amide groups, expected around $1650 \mathrm{~cm}^{-1}$ (amide I) was not detected. The lack of this distinctive peak could be possibly ascribed to a small number of PEG chains grafted to the sh-SWNTs.

${ }^{1} \mathrm{H}$ NMR spectra in $\mathrm{CDCl}_{3}$ (room temperature) of the soluble samples show a broad resonance centered at $\delta=3.64$ which can be assigned to the methylene groups of PEG chains. The broadening of PEG resonances could be due, at least in part, to the polydispersed nature of the PEG-amine reagent or to residual paramagnetic metal catalysts used for NTs synthesis. In addition, the broadening could result from the statistical distribution of the addends on the NTs that are mixtures of NTs with different length and diameters as pointed out earlier.

Unfortunately, ${ }^{13} \mathrm{C}$ NMR spectra did not show any meaningful signal for the NTs carbons or the amide carbonyl carbons. This is likely due to both broadening and low sample concentration.

Figure 1 shows the Raman spectrum of PEG-sh-SWNT in $\mathrm{CHCl}_{3}\left(\lambda_{\text {exc }}=488 \mathrm{~nm}\right)$ with a major peak at $1592 \mathrm{~cm}^{-1}$ assigned to the tangential mode transition. This indicates that the basic NT's features are retained also in the functionalized samples, although the peak related to the 
radial mode was not detected. This is probably due to its intrinsic low intensity at the excitation wavelength, as previously reported for a nearby $514 \mathrm{~nm}$ excitation line. ${ }^{47}$ In addition, we have not observed a strong luminescence background, which usually interferes with Raman scattering, perhaps indicating a sufficient purity of the synthesized PEG-sh-SWNTs. Interestingly, the peak of the tangential mode shows a shoulder at $1567 \mathrm{~cm}^{-1}$ suggesting that the solubilized material in $\mathrm{CHCl}_{3}$ is mostly present as bundles of NTs. ${ }^{47}$

An indirect confirmation of the latter observation comes from the TEM image of Figure 2a in which a bundle of sh-SWNTs, cut in a staircase-like shape and a long truncated NT, is shown. The other NTs of the bundle terminate as a flute a bec. Although we could not identify the exact grafting sites, it is reasonable to assume that on these sharp edges, possibly rich of carboxylic groups, the PEG chains would link through an amide bond. Such structures were not observed for the pristine SWNTs prior to the cutting and etching treatment. Figure $2 \mathrm{~b}$ shows one of the TEM images we registered for pristine SWNTs where at the centre it is possible to observe a NT terminating with the expected hemifullerene cup.

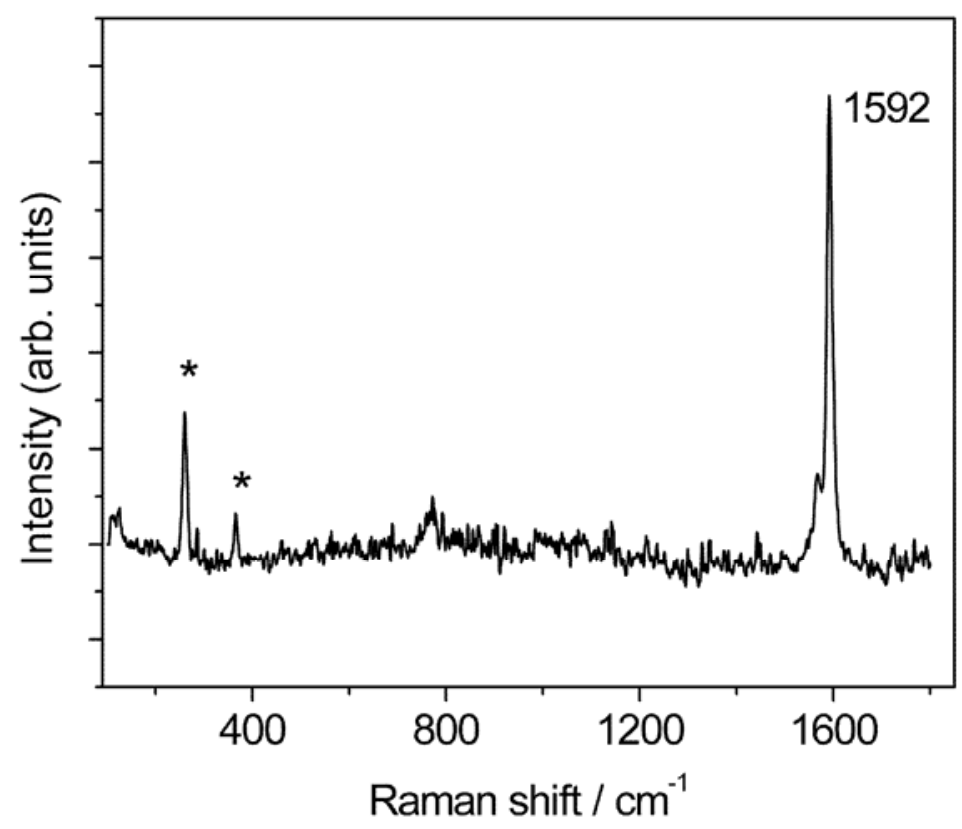

Figure 1. Raman spectrum (exciting line at $488 \mathrm{~nm}$ ) of a solution of sh-SWNT-PEG in chloroform; the peaks labeled $\left(^{*}\right)$ are due to chloroform. The baseline was corrected for the luminescence background.

Carbon nanotubes have been considered for their non-linear optical properties and, in particular, for their non-linear transmission properties which may find application in optical limiting devices. ${ }^{8,9,22,48,49}$ Figure 3 shows the non linear transmission measurements of a PEG-shSWNTs solution and of a suspension of pristine SWNTs in $\mathrm{CHCl}_{3}$ obtained by using 9 ns pulses at $532 \mathrm{~nm}$ from a Nd:YAG laser. Contrary to the behaviour of soluble NTs prepared by another 
group, ${ }^{22}$ PEG-sh-SWNTs show a better optical limiting response than that displayed by a suspension of SWNTs. This result suggests that different conditions of cutting, etching and functionalization may induce important changes of the properties of soluble NTs samples. The data we recorded for the PEG-sh-SWNTs recall those found for reverse saturable absorber molecules, such as the fullerenes ${ }^{50}$ or phthalocyanines. ${ }^{51}$
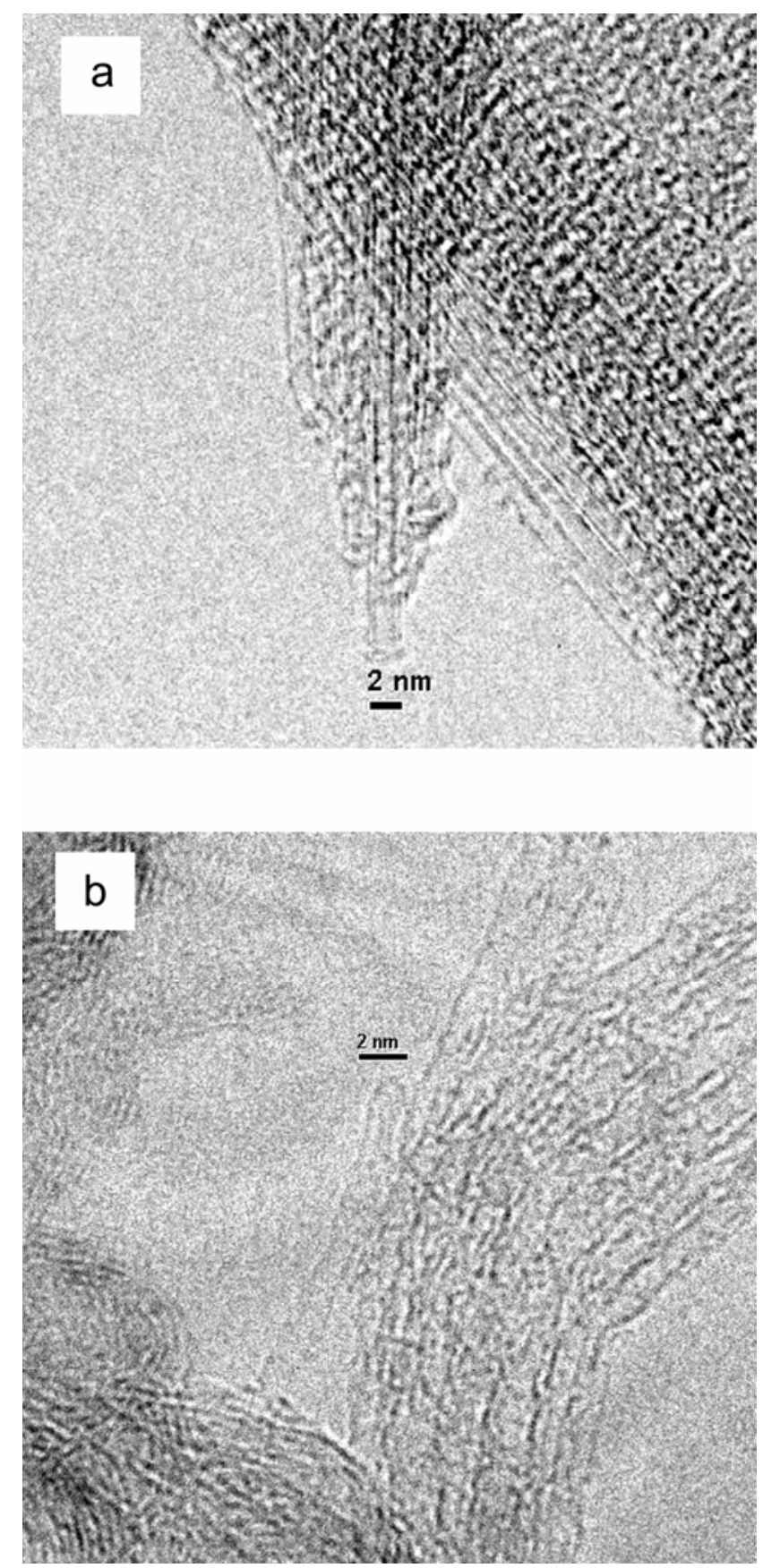

Figure 2. TEM images of a) sh-SWNTs (see text) and b) pristine SWNTs showing a single nanotube with the hemifullerene terminal cup at the center of the image. 
The data for the SWNTs suspension recall the behavior of non-linear scattering systems, such as carbon black or gold clusters. ${ }^{52}$ The hypothesis that the dominant mechanism of limitation is different for the suspension and for the solubilized NTs and that, particularly for the latter, there is a dominant excited state absorption, was already suggested by Sun and coworkers. ${ }^{22}$ Further investigations are however needed to confirm the above hypothesis. It is worth mentioning, moreover, that the suspensions of pristine SWNTs were very unstable when irradiated with highenergy laser pulses. After a few pulses, the suspended NTs coagulate into macroscopic aggregates that eventually sediment in the cuvette. On the other hand, the PEG-sh-SWNTs solutions were very stable also after prolonged irradiation. This could be probably ascribed to PEG chains that contrast bundle-to-bundle interactions thus preventing macroscopic NTs aggregation.

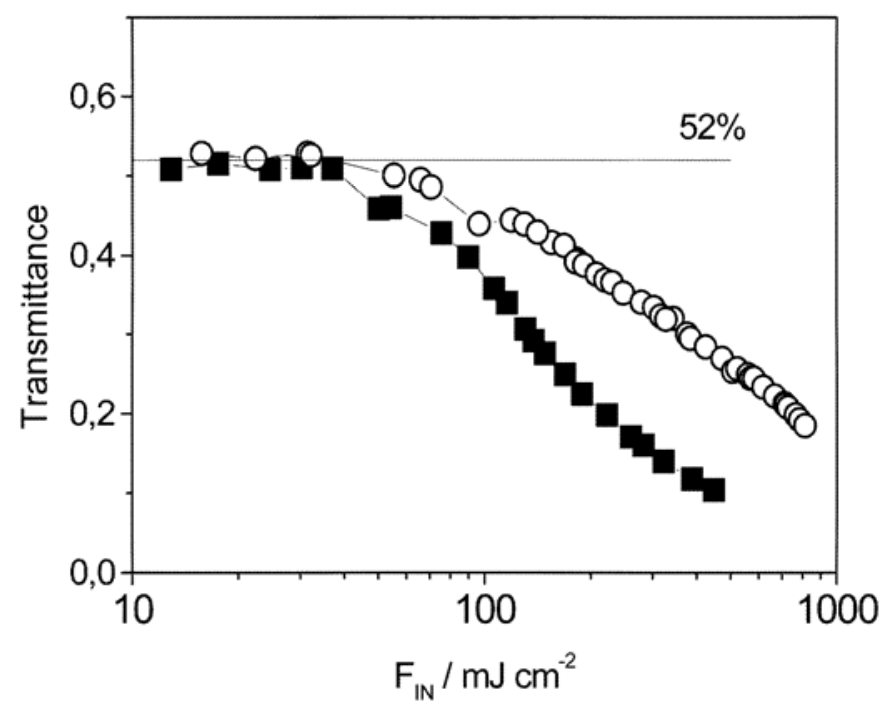

Figure 3. Non-linear transmittance of sh-SWNT-PEG (solid squares) and of suspended SWNT (open circles) in chloroform.

\section{Conclusions}

sh-SWNTs were functionalized with hydrophilic PEG chains under standard amidation reaction conditions. The functionalization possibly involves the sh-SWNTs-bonded carboxylic groups although an unambiguous confirmation of the proposed amide linkage is still pending. However, proton NMR, Raman spectroscopy and the fact that blends of SWNT-CO ${ }_{2} \mathrm{H}$ with PEG-amine did not give any soluble material represent complementary evidence for the soluble samples being functionalized NTs. PEG-sh-SWNTs are moderately soluble in THF and chlorinated 
hydrocarbons. For instance stock solutions of PEG-sh-SWNTs in $\mathrm{CHCl}_{3}$ (ca. $1 \mathrm{mg} / \mathrm{ml}$ ) are stable for weeks without any apparent precipitation. Finally, returning to our initial motivation, the optical limiting experiments reported here show that solutions of PEG-sh-SWNTs in $\mathrm{CHCl}_{3}$ show a better optical limiting response and higher stability than that displayed by a suspension of SWNTs in the same solvent. The data for the PEG-sh-SWNTs recall those recorded for reverse saturable absorber molecules, such as the fullerenes or phthalocyanines, indicating that excited state absorption may play a relevant role in the mechanism of optical limitation for NTs solutions. Further investigations in this direction are underway in our laboratories.

\section{Experimental Section}

Instrumentation and materials. Original SWNTs were purchased from Tubes@Rice, Houston, TX, USA. Poly(ethylene glycol) monoamine (PEG-amine, molecular weight $=2000$ ) was purchased from SunBio, Walnut Creek, CA, USA. Filtrations were performed on Millipore VCTP polycarbonate membranes $\left(0.1 \mu \mathrm{m}\right.$ pore size). $\mathrm{SOCl}_{2}$ and all solvents used for the workup of PEG-sh-SWNTs were distilled prior to use. Those used for non linear transmission measurements were spectrophotometric-grade solvents. For cutting SWNTs a 300W ultrasonic cleaning bath, operating at 25kHz, was used. NMR spectra were recorded on a Bruker AC 250 (5.9 T, 250.1 MHz for ${ }^{1} \mathrm{H}$ ). Raman spectra were registered on a Jobin Yvon S3000 equipped with a CCD detector (Astromed mod. 2200) and a mixed Ar-Kr laser (Coherent mod. Innova 70 Spectrum). IR spectra were recorded on a Perkin-Elmer $1720 \mathrm{X}$. Non linear transmittance measurements were carried out by using 9 ns pulses of a Nd:YAG laser (Quantel YG 981E) duplicated at $532 \mathrm{~nm}$. HR-TEM images were obtained with a JEM 3010 (JEOL) operating at 300 $\mathrm{kV}$.

Synthesis of PEG-sh-SWNTs. Commercial SWNTs were cut and etched according to a previously reported procedure. ${ }^{43}$ Sonication, during the cutting protocol, was reduced to $8 \mathrm{~h}$ in order to avoid excessive degradation of NTs. The quality of the isolated sh-SWNTs was checked by Raman spectroscopy and TEM analysis. Acid oxidative cutting and etching processes produces carboxylic groups on SWNTs. Conversion of these groups to the corresponding acid chlorides (SWNT-COCl) was achieved by $\mathrm{SOCl}_{2}$ treatment as previously reported.16 SWNTCOCl (56 mg) and PEG-amine (795 mg) were gently stirred at $90{ }^{\circ} \mathrm{C}$ for $96 \mathrm{~h}$ under a dry nitrogen atmosphere. The black solid, obtained after cooling the viscous reaction mixture, was repeatedly treated with water $(5 \times 20 \mathrm{ml})$ under sonication in order to remove excess of PEGamine and its potential degradation products. After each treatment, the sample was centrifuged at $4000 \mathrm{rpm}$ for $15 \mathrm{~min}$. and the supernatant aqueous dispersion filtered through a $0.1 \mu \mathrm{m}$ membrane. The solid sediment on the membrane was further washed with water and added to the combined solid residues recovered after centrifugation. The overall solid material was then dispersed in tetrahydrofuran (THF, $20 \mathrm{ml}$ ), sonicated for $30 \mathrm{~min}$. and centrifuged. The solid 
residue was separated from the supernatant and further washed with THF $(3 \times 5 \mathrm{ml})$. The combined THF washings were evaporated at reduced pressure and the solid residue, dissolved in the minimum amount of THF, was precipitated upon addition of cyclohexane (5 ml) and centrifuged. The product was then washed with cyclohexane $(3 \times 5 \mathrm{ml})$, diethyl ether and dried in vacuum affording $7.5 \mathrm{mg}$ of a black solid material.

\section{Acknowledgments}

The authors thank G. Marcolongo for technical assistance. This work was supported by CNR (Agenzia 2000) and MIUR (Contract n. 2002032171).

\section{References}

1. Iijima, S. Nature 1991, 354, 56.

2. Dresselhaus, M. S.; Dresselhaus, G.; Avouris, P. Carbon Nanotubes: Synthesis, Structure, Properties, and Applications; Springer: Heidelberg, 2001, Vol. 80.

3. Baughman, R. H.; Zakhidov, A. A.; de Heer, W. A. Science 2002, 297, 787.

4. Rao, C. N. R.; Satishkumar, B. C.; Govindaraj, A.; Nath, M. Chem. Phys. Chem. 2001, 2, 78.

5. Avouris, P. Chem. Phys. 2002, 281, 429.

6. Andrews, R.; Jacques, D.; Rao, A. M.; Rantell, T.; Derbyshire, F.; Chen, Y.; Chen, J.; Haddon, R. C. Appl. Phys. Lett. 1999, 75, 1329.

7. Wong, E. W.; Sheehan, P. E.; Lieber, C. M. Science 1997, 277, 1971.

8. Chen, P.; Wu, X.; Sun, X.; Lin, J.; Ji, W.; Tan, K. L. Phys. Rev. Lett. 1999, 82, 2548.

9. Vivien, L.; Lancon, P.; Riehl, D.; Hache, F.; Anglaret, E. Carbon 2002, 40, 1789.

10. Ma, Y. C.; Xia, Y. Y.; Zhao, M. W.; Ying, M. J. Phys. Rev. B 2002, 6515, NIL_969.

11. Krstic, V.; Duesberg, G. S.; Muster, J.; Burghard, M.; Roth, S. Chem. Mater. 1998, 10, 2338.

12. Ausman, K. D.; Piner, R.; Lourie, O.; Ruoff, R. S.; Korobov, M. J. Phys. Chem. B 2000, 104, 8911.

13. Steuerman, D. W.; Star, A.; Narizzano, R.; Choi, H.; Ries, R. S.; Nicolini, C.; Stoddart, J. F.; Heath, J. R. J. Phys. Chem. B 2002, 106, 3124.

14. Star, A.; Stoddart, J. F.; Steuerman, D.; Diehl, M.; Boukai, A.; Wong, E. W.; Yang, X.; Chung, S.-W.; Choi, H.; Heath, J. R. Angew. Chem., Int. Ed. 2001, 40, 1721.

15. O'Connell, M. J.; Boul, P.; Ericson, L. M.; Huffman, C.; Wang, Y.; Haroz, E.; Kuper, C.; Tour, J.; Ausman, K. D.; Smalley, R. E. Chem. Phys. Lett. 2001, 342, 265.

16. Chen, R. J.; Zhan, Y. G.; Wang, D. W.; Dai, H. J. J. Am. Chem. Soc. 2001, 123, 3838.

17. Sun, Y.; Wilson, S. R.; Schuster, D. I. J. Am. Chem. Soc. 2001, 123, 5348.

18. Chen, J.; Hamon, M. A.; Hu, H.; Chen, Y. S.; Rao, A. M.; Eklund, P. C.; Haddon, R. C. Science 1998, 282, 95. 
19. Hamon, M. A.; Chen, J.; Hu, H.; Chen, Y. S.; Itkis, M. E.; Rao, A. M.; Eklund, P. C.; Haddon, R. C. Advanced Materials 1999, 11, 834.

20. Hamon, M. A.; Hu, H.; Bhowmik, P.; Niyogi, S.; Zhao, B.; Itkis, M. E.; Haddon, R. C. Chem. Phys. Lett. 2001, 347, 8.

21. Zhao, B.; Hu, H.; Niyogi, S.; Itkis, M. E.; Hamon, M. A.; Bhowmik, P.; Meier, M. S.; Haddon, R. C. J. Am. Chem. Soc. 2001, 123, 11673.

22. Riggs, J. E.; Walker, D. B.; Carroll, D. L.; Sun, Y. P. J. Phys. Chem. B 2000, 104, 7071.

23. Riggs, J. E.; Guo, Z. X.; Carroll, D. L.; Sun, Y. P. J. Am. Chem. Soc. 2000, 122, 5879.

24. Sun, Y. P.; Huang, W. J.; Lin, Y.; Fu, K. F.; Kitaygorodskiy, A.; Riddle, L. A.; Yu, Y. J.; Carroll, D. L. Chem. Mater. 2001, 13, 2864.

25. Sano, M.; Kamino, A.; Okamura, J.; Shinkai, S. Langmuir 2001, 17, 5125.

26. Banerjee, S.; Wong, S. S. Nano Letters 2002, 2, 195.

27. Huang, W. J.; Taylor, S.; Fu, K. F.; Lin, Y.; Zhang, D. H.; Hanks, T. W.; Rao, A. M.; Sun, Y. P. Nano Letters 2002, 2, 311.

28. Pompeo, F.; Resasco, D. E. Nano Letters 2002, 2, 369.

29. Huang, W. J.; Lin, Y.; Taylor, S.; Gaillard, J.; Rao, A. M.; Sun, Y. P. Nano Letters 2002, 2, 231.

30. Basiuk, E. V.; Basiuk, V. A.; Banuelos, J. G.; Saniger Blesa, J. M.; Pokrovskiy, V. A.; Gromovoy, T. Y.; Mischanchuk, A. V.; Mischanchuk, B. G. J. Phys. Chem. B 2002, 106, 1588.

31. Huang, W. J.; Fernando, S.; Allard, L. F.; Sun, Y. P. Nano Letters 2003, 3, 565.

32. Chiu, P. W.; Duesberg, G. S.; Dettlaff Weglikowska, U.; Roth, S. Appl. Phys. Lett. 2002, 80, 3811.

33. Shim, M.; Kam, N. W. S.; Chen, R. J.; Li, Y. M.; Dai, H. J. Nano Letters 2002, 2, 285.

34. Fu, K. F.; Huang, W. J.; Lin, Y.; Zhang, D. H.; Hanks, T. W.; Rao, A. M.; Sun, Y. P. Journal of Nanoscience and Nanotechnology 2002, 2, 457.

35. Sun, Y. P.; Fu, K. F.; Lin, Y.; Huang, W. J. Acc. Chem. Res. 2002, 35, 1096.

36. Hill, D. E.; Lin, Y.; Rao, A. M.; Allard, L. F.; Sun, Y. P. Macromolecules 2002, 35, 9466.

37. Lin, Y.; Taylor, S.; Huang, W. J.; Sun, Y. P. J. Phys. Chem. B 2003, 107, 914.

38. Della Negra, F.; Meneghetti, M.; Menna, E. Fuller. Nanotub. Car. N. 2002, 11, 25.

39. Hirsch, A. Angew. Chem., Int. Ed. 2002, 41, 1853.

40. Niyogi, S.; Hamon, M. A.; Hu, H.; Zhao, B.; Bhowmik, P.; Sen, R.; Itkis, M. E.; Haddon, R. C. Acc. Chem. Res. 2002, 35, 1105.

41. Georgakilas, V.; Kordatos, K.; Prato, M.; Guldi, D. M.; Holzinger, M.; Hirsch, A. J. Am. Chem. Soc. 2002, 124, 760.

42. Bauschlicher, C. W. Chem. Phys. Lett. 2000, 322, 237.

43. Liu, J.; Rinzler, A. G.; Dai, H. J.; Hafner, J. H.; Bradley, R. K.; Boul, P. J.; Lu, A.; Iverson, T.; Shelimov, K.; Huffman, C. B.; Rodriguez-Macias, F.; Young-Seok, S.; Lee, T. R.; Colbert, D. T.; Smalley, R. E. Science 1998, 280, 1253. 
44. Harris, J. M.; Zalipsky, S. Chemistry and Biological Applications of Polyethylene Glycol; ACS Books: Washington, D.C., 1997.

45. Pimenta, M. A.; Marucci, A.; Brown, S. D. M.; Matthews, M. J.; Rao, A. M.; Eklund, P. C.; Smalley, R. E.; Dresselhaus, G.; Dresselhaus, M. S. J. Mater. Res. 1998, 13, 2396.

46. Rao, A. M.; Richter, E.; Bandow, S.; Chase, B.; Eklund, P. C.; Williams, K. A.; Fang, S.; Subbaswamy, K. R.; Menon, M.; Thess, A.; Smalley, R. E.; Dresselhaus, G.; Dresselhaus, M. S. Science 1997, 275, 187.

47. Rao, A. M.; Chen, J.; Richter, E.; Schlecht, U.; Eklund, P. C.; Haddon, R. C.; Venkateswaran, U. D.; Kwon, Y. K.; Tomanek, D. Phys. Rev. Lett. 2001, 86, 3895.

48. Mishra, S. R.; Rawat, H. S.; Mehendale, S. C.; Rustagi, K. C.; Sood, A. K.; Bandyopadhyay, R.; Govindaraj, A.; Rao, C. N. R. Chem. Phys. Lett. 2000, 317, 510.

49. Vivien, L.; Anglaret, E.; Riehl, D.; Hache, F.; Bacou, F.; Andrieux, M.; Lafonta, F.; Journet, C.; Goze, C.; Brunet, M.; Bernier, P. Opt. Commun. 2000, 174, 271.

50. Signorini, R.; Meneghetti, M.; Bozio, R.; Maggini, M.; Scorrano, G.; Prato, M.; Brusatin, G.; Innocenzi, P.; Guglielmi, M. Carbon 2000, 38, 1653.

51. Perry, J. W.; Mansour, K.; Lee, I. Y. S.; Wu, X. L.; Bedworth, P. V.; Chen, C. T.; Ng, D.; Marder, S. R.; Miles, P.; Wada, T.; Tian, M.; Sasabe, H. Science 1996, 273, 1533.

52. Francois, L.; Mostafavi, M.; Belloni, J.; Delouis, J. F.; Delaire, J.; Feneyrou, P. J. Phys. Chem. B 2000, 104, 6133. 